\title{
A Fully-Implicit High-Order System Thermal-Hydraulics Model for Advanced Reactor Safety Analyses
}

\author{
Rui Hu
}

\begin{abstract}
An advanced system analysis tool is being developed for advanced reactor safety analysis. This paper describes the underlying physics and numerical models used in the code, including the governing equations, the stabilization schemes, the high-order spatial and temporal discretization schemes, and the Jacobian Free Newton Krylov solution method. The effects of the spatial and temporal discretization schemes are investigated. Additionally, a series of verification test problems are presented to confirm the high-order schemes. It is demonstrated that the developed system thermal-hydraulics model can be strictly verified, and that it performs very well for a wide range of flow problems with high accuracy, efficiency, and minimal numerical diffusions.
\end{abstract}

KEYWORDS

high-order, fully-implicit, FEM, system thermal-hydraulics

\section{INTRODUCTION}

System thermal-hydraulics (STH) remains as one of the major disciplines essential for the design and operation of nuclear systems [1]. Many system analysis codes, such as RELAP5, CATHARE and SAS4A/SASSYS-1, have been developed since the early 1970s and successfully applied for the design, license, and operational analysis of the nuclear power plants. Although these codes have achieved a high level maturity, they have not taken full advantage of the rapid expansion in computing power and advances in numerical methods over the past two decades. Most existing reactor system analysis codes were developed based on low-order numerical schemes in both space and time. However, it is very challenging to rely on the first-order methods to accurately model the sudden disturbances or wave propagations, as commonly observed or expected in reactor transients, without the concerns of numerical instability and numerical diffusion. For example, the semi-implicit methods with the staggered grid mesh and the donor cell upwind method are commonly used in existing codes for simplicity and stableness. However, these schemes are highly diffusive and not desirable for the conditions mentioned above.

With advances in numerical techniques and software engineering, there has been a renewed interest in advanced STH code developments such as RELAP-7 [2] and CATHARE-3 [3] for advanced physical and numerical modeling of two-phase flows. Additionally, research in high-order numerical schemes for system simulation of two-phase flow is also of increasing interest [4,5,6]. High-resolution spatial discretization schemes provide high order spatial accuracy in smooth regions and capture sharp spatial discontinuity without nonphysical spatial oscillations. High-order temporal discretization and the fully implicit schemes improve the temporal accuracy, as well as allow for the use of large time step sizes for efficiency. 
Under the U.S. Department of Energy (DOE) Nuclear Energy Advanced Modeling and Simulation (NEAMS) program, a system analysis module (SAM) [7,8] is being developed at Argonne National Laboratory for advanced reactor system analysis. It focuses on the modeling of the components and systems that represent typical features of advanced reactor concepts such as SFRs (sodium fast reactors), LFRs (lead-cooled fast reactors), and FHRs (fluoride-salt-cooled high temperature reactors). These advanced concepts are distinguished from light-water reactors in their use of single-phase, low-pressure, high-temperature, and low Prandtl number (sodium and lead) coolants. This simple yet fundamental change has significant impacts on core and plant design, the types of materials used, component design and operation, fuel behavior, and the significance of the fundamental physics in play during transient plant simulations.

To facilitate the code development, SAM utilizes an object-oriented application framework (MOOSE [9]), and its underlying meshing and finite-element library (libMesh [10]) and linear and non-linear solvers (PETSc [11]), to leverage the modern advanced software environments and numerical methods. SAM aims to solve the tightly-coupled physical phenomena including fission reaction, heat transfer, fluid dynamics, and thermal-mechanical response in the advanced reactor structures, systems and components with high accuracy and efficiency. The high-order spatial discretization schemes, fully-implicit and highorder time integration schemes, and the advanced solution method (such as the Jacobian-free NewtonKrylov (JFNK) method) are the key aspects in developing an accurate and computationally efficient model in SAM.

The initial SAM development efforts focused on the modeling and simulation capabilities of single-phase fluid dynamics and heat transfer. This paper briefly discusses the underlying one-dimensional finiteelement flow formulation for incompressible but thermally expandable flow and the stabilization schemes. Additionally, the high-order spatial discretization schemes, the high-order temporal discretization scheme, and the JFNK solution method are discussed. The effects of the spatial and temporal discretization schemes are examined by series of verification tests, some of which are utilized to confirm the high-order numerical convergence rates.

\section{SAM PHYSICS AND NUMERICAL MODELS}

\subsection{Governing Equations}

SAM employs a one-dimensional transient model for single-phase incompressible but thermally expandable flow. The governing equations consist of the continuity equation, momentum equation, and energy equation. A three dimensional module is also under development to model the multi-dimensional flow and thermal stratification in the upper plenum or the cold pool of the SFR reactor vessel. Additionally, a subchannel module is being developed for fuel assembly modeling. Both the 3-D module and the sub-channel module will require additional momentum conservation equations. However, they are not the essential modeling capabilities for a system code and are still under development, thus will not be further discussed in this paper.

The transport equations for one-dimensional, single-phase flow can be described by the following set of PDEs. The mass, momentum, and energy conservation equations are closed by the equation of state of the fluid and the other constitutive equations for friction and heat transfer models. After the simplifications and applying the continuity equation into the momentum and energy equations, the set of governing equations can be written in the conservative form (Eq.-1) or in the non-conservative form (Eq.-2). 


$$
\begin{aligned}
& \frac{\partial \rho}{\partial t}+\frac{\partial(\rho u)}{\partial z}=0 \\
& \frac{\partial(\rho u)}{\partial t}+\frac{\partial(\rho u u+p)}{\partial z}=-\rho g-\frac{f}{D_{e}} \frac{\rho u|u|}{2} \\
& \frac{\partial(\rho H)}{\partial t}+\frac{\partial(\rho u H)}{\partial z}=q^{\prime \prime \prime} \\
& \rho=\rho(p, T)
\end{aligned}
$$

In which $f$ : the friction coefficient; $D_{e}$ : equivalent hydraulic diameter. When considering the convection heat flux from solid surface $q_{s}^{\prime \prime}, q^{\prime \prime \prime}=q_{s}^{\prime \prime} P_{h} / A_{c}$, where $P_{h}$ and $A_{c}$ respectively denote heated perimeter and cross-sectional area of the coolant channel.

$$
\begin{aligned}
& \frac{\partial \rho}{\partial t}+\frac{\partial(\rho u)}{\partial z}=0 \\
& \rho \frac{\partial u}{\partial t}+\rho u \frac{\partial u}{\partial z}=-\frac{\partial p}{\partial z}-\rho g-\frac{f}{D_{e}} \frac{\rho u|u|}{2} \\
& \rho C_{p} \frac{\partial T}{\partial t}+\rho C_{p} u \frac{\partial T}{\partial z}=q^{\prime \prime \prime} \\
& \rho=\rho(p, T)
\end{aligned}
$$

In which $C_{p}$ : the specific heat, $C_{p}=\frac{\partial H}{\partial T}$.

To solve the above sets of PDEs, two out of three variables of density, pressure, and temperature (or total energy) must be selected as the state variables, along with the velocity (or mass flux) to compose the three state variables for the system of equations. The primitive variable (or pressure) based formulation, in which the state variables are pressure $(p)$, velocity $(u)$, and temperature $(T)$, is developed in SAM. Although the conservative variable (or density) based formulation has the advantages in the applications of compressible flow such as the capability to capture shock waves, the primitive variable based FEM formulation is more suitable for incompressible or nearly incompressible flows, such as the fluid flow in SFRs, LFRs, or FHRs. With the primitive variable approach, using the integral equations in the conservative form (Eq.-1) will still ensure the conservation laws of the fluid equations.

Finite element analysis of incompressible flows requires stabilization to avoid the potential numerical instabilities. The Streamline-Upwind/Petrov-Galerkin (SUPG) and the Pressure-Stabilizing/PetrovGalerkin (PSPG) scheme are implemented in SAM to resolve the numerical instability issues. The details of the single-phase incompressible thermally expandable flow model and the stabilization schemes can be found in Reference [12]. A review of stabilized finite element formulations for incompressible flow, including the SUPG and PSPG schemes, can be found in Ref. [13].

The weak forms of the stabilization schemes for incompressible flow can be derived as:

$$
\begin{gathered}
\left(\frac{\partial \rho}{\partial t}+\frac{\partial(\rho u)}{\partial z}, \psi\right)+\left(\frac{\partial \rho}{\partial t}+\rho u \frac{\partial u}{\partial z}+\frac{\partial p}{\partial z}+\rho g+\frac{f}{D_{e}} \frac{\rho u|u|}{2}, \tau_{P S P G} * \nabla \psi\right)=0 \\
\left(\frac{\partial \rho u}{\partial t}+\frac{\partial \rho u u}{\partial z}+\frac{\partial p}{\partial z}+\rho g+\frac{f}{D_{e}} \frac{\rho u|u|}{2}, \psi\right) \\
+\left(\rho \frac{\partial u}{\partial t}+\rho u \frac{\partial u}{\partial z}+\frac{\partial p}{\partial z}+\rho g+\frac{f}{D_{e}} \frac{\rho u|u|}{2}, \tau_{S U P G} * \nabla \psi\right)=0 \\
\left(\frac{\partial \rho H}{\partial t}+\frac{\partial \rho u H}{\partial z}-q^{\prime \prime \prime}, \psi\right)+\left(\rho C_{p} \frac{\partial T}{\partial t}+\rho C_{p} u \frac{\partial T}{\partial z}-q^{\prime \prime \prime}, \tau_{S U P G} * \nabla \psi\right)=0
\end{gathered}
$$


in which $\psi$ is the test function; $\tau_{P S P G}$ and $\tau_{S U P G}$ are the stabilization parameters that weights the perturbations; and $(f, \psi)=\int_{\Omega} \psi * f d \Omega$, is an expression of the volume integral. Note that the regular residuals of all conservation equations are calculated based on the conservative form while the stabilization terms are calculated based on the non-conservative form. This formulation not only ensures the conservation laws, but also is easy to be implemented.

In SAM, heat structures model the heat conduction inside the solids and permit the modeling of heat transfer at the interfaces between solid and fluid components. Heat structures are represented by onedimensional or two-dimensional heat conduction in Cartesian or cylindrical coordinates. Temperaturedependent thermal conductivities and volumetric heat capacities can be provided in tabular or functional form either from built-in or user-supplied data. The modeling capabilities of heat structures can be used to predict the temperature distributions in solid components such as fuel pins or plates, heat exchanger tubes, and pipe and vessel walls, as well as to calculate the heat flux conditions for fluid components.

The thermal conduction inside the solid structures is governed by a diffusion equation:

$$
\rho C_{p} \frac{\partial T}{\partial t}-\nabla(k \nabla T)-Q^{\prime \prime \prime}=0
$$

Where $k$ is the solid thermal conductivity, and $Q^{\prime \prime \prime}$ is the volumetric internal heat source in the solid. It can be discretized in both Cartesian and cylindrical coordinates. The types of boundary conditions (BC) include: (1) Dirichlet $\mathrm{BC}, T=T_{0}$; (2) Neumann $\mathrm{BC}, k \nabla T=q_{0}$ "; or (3) convective $\mathrm{BC}:-k \nabla T=h$. $\left(T-T_{\text {fluid }}\right)$. A more in-depth discussion of the conjugate heat transfer modeling in SAM can be found in Ref. [14].

\subsection{Stabilization Parameters $\tau_{P S P G}$ and $\tau_{S U P G}$}

In SUPG and PSPG stabilization schemes, additional stabilization terms are added to the standard Galerkin formulation of the fluid equations. Judicious selection of the stabilization parameters, $\tau_{P S P G}$ and $\tau_{S U P G}$, plays a key role in determining the stability and accuracy of the formulations. Various $\tau$ formulations were proposed in the literature. The stabilization parameters often involve a measure of the local length scale and other parameters such as the local Reynolds number and Courant numbers. For onedimensional space-time formulation, the formulation of the stabilization parameters can be defined as:

$$
\begin{aligned}
& \tau=\left[\left(\frac{2}{\Delta t}\right)^{2}+\left(\frac{2|u|}{h}\right)^{2}+\left(\frac{4 v}{h^{2}}\right)^{2}\right]^{-1 / 2} \\
& \tau_{S U P G}=u \cdot \tau \\
& \tau_{P S P G}=\tau(\mathrm{U})
\end{aligned}
$$

Where $h$ is the "element length", $u$ is local velocity, $\Delta t$ is the time step size, $v$ is the kinematic viscosity, and $U$ is a global scaling velocity. If $U$ were not defined in the simulation, the local velocity would be used. This formulation is modified based on the one-dimensional space-time formulation given in Ref. [15]. For one-dimensional fluid model in reactor safety analysis, large elements are commonly used, where $\left(\frac{2|u|}{h}\right) \gg\left(\frac{4 v}{h^{2}}\right)$ or $\frac{|\mathrm{u}| \mathrm{h}}{v} \gg 2$. Therefore, the stabilization parameters can be simplified as:

$$
\begin{aligned}
& \tau_{S U P G}=u \cdot\left[\left(\frac{2}{\Delta t}\right)^{2}+\left(\frac{2|u|}{h}\right)^{2}\right]^{-1 / 2} \\
& \tau_{P S P G}=\left[\left(\frac{2}{\Delta t}\right)^{2}+\left(\frac{2|U|}{h}\right)^{2}\right]^{-1 / 2}
\end{aligned}
$$

Additionally, an upper bound and lower bound were defined for $\tau_{P S P G}$ to ensure the robustness of the stabilization scheme under extreme flow conditions. 


\subsection{Spatial Discretization Errors}

In SAM, both linear elements (EDGE2 in 1-D and QUAD4 in 2-D) and the second-order elements (EDGE3 and QUAD9) are available for use in the finite-element discretization of fluid flow and solid structures. For first-order elements using piece-wise linear Language shape functions, the trapezoidal rule is recommended for the numerical integration; while the Gaussian quadrature rule is recommended for second-order elements (with second-order Language shape functions) in SAM. In one-D analysis,

$$
\begin{aligned}
& \text { Trapezoidal rule: } \int_{a}^{b} f(x) d x=(b-a) \frac{[f(a)+f(b)]}{2} \\
& \text { Gaussian quadrature rule: } \int_{a}^{b} f(x) d x=\sum_{q p} f\left(x_{q p}\right) w_{q p}
\end{aligned}
$$

In which $x_{q p}$ is the quadrature point, and $w_{q p}$ is the weight. In SAM, the Gauss-Legendre quadrature is used (through MOOSE and LibMesh); and the quadrature points and weights are well defined.

In One-Dimension, Trapezoid formula with an interval h gives error of the order $O\left(h^{2}\right)$. On the other hand, the Gaussian quadrature rule can exactly integrate polynomials of order $2 n-1$ with $n$ quadrature points. However, the error can be difficult to estimate as it depends on the $2 n$ order derivative. The error bound is [16],

$$
\text { Error }=\int_{a}^{b} f(x) d x-\sum_{q p} f\left(x_{q p}\right) w_{q p}=\frac{(b-a)^{2 n+1}(n !)^{4}}{(2 n+1)[(2 n) !]^{3}} f^{(2 n)}(\xi), a<\xi<b .
$$

It is can be concluded that SAM spatial discretization scheme is at least second-order accurate with the first-order elements, and could have exponential convergence rates with the second-order elements for continuous problems.

\subsection{Temporal Discretization Errors}

SAM, through MOOSE, supports a number of standard time integration methods such as the explicit Euler, implicit Euler (or backward Euler), and BDF2 (backward differentiation formula $-2^{\text {nd }}$ order) method, Crank-Nicolson, and Runge-Kutta methods. For most reactor applications, we recommend to use the implicit Euler or BDF2 methods with SAM.

The backward differentiation formula (BDF) is a family of implicit methods for the numerical integration of ordinary differential equations. They are linear multistep methods that, for a given function and time, approximate the derivative of that function using information from already computed times, thereby increasing the accuracy of the approximation. Note that the first order method of this family, BDF1, is equivalent to the backward Euler method. For a time-step-size $\Delta t$, applying the BDF methods to the ordinary differential equation:

$$
\frac{\partial u}{\partial t}=f(u, t)
$$

would result in:

$$
\begin{aligned}
& f\left(u^{n+1}, t^{n+1}\right)=\frac{u^{n+1}-u^{n}}{\Delta t}+O(\Delta t), \text { Backward Euler or BDF1; } \\
& f\left(u^{n+1}, t^{n+1}\right)=\frac{\frac{3}{2} u^{n+1}-2 u^{n}+\frac{1}{2} u^{n-1}}{\Delta t}+O\left(\Delta t^{2}\right), B D F 2 .
\end{aligned}
$$

It can be conclude that SAM temporal discretization can be second-order accurate when using the BDF2 scheme. 


\subsection{Solution Methods}

The Jacobian-Free Newton Krylov (JFNK) solution method is used to the above equation system. The JFNK method is a multi-level approach, the outer Newton's iterations (nonlinear solver) and inner Krylov subspace methods (linear solver), in solving large nonlinear systems. The concept of 'Jacobian-free' is proposed, because deriving and assembling large Jacobian matrices could be difficult and expensive. The JFNK method has become an increasingly popular option for solving large nonlinear equation systems and multi-physics problems, as observed in a number of different disciplines [17]. One great feature of JFNK is that all the unknowns are solved simultaneously in a fully coupled fashion. This solution scheme avoids the errors from operator splitting and is especially suitable for conjugate heat transfer problems in which the heat conduction in the solid is tightly coupled with the fluid flow.

Assuming that the FEM discretized equations form a system of discretized nonlinear equations:

$$
F(u)=0
$$

Where $\mathrm{F}$ represents the nonlinear equation system and $\mathrm{u}$ is the solution vector. Newton's method will be like solving:

$$
J\left(u^{k}\right) \delta u^{k}=-F\left(u^{k}\right)
$$

given $u^{0}$, In which, $u^{k+1}=u^{k}+\delta u^{k}, \mathrm{~J}$ is the associated Jacobian matrix and $J\left(u^{k}\right)=F^{\prime}\left(u^{k}\right)=$ $\left.\frac{d F(u)}{d u}\right|_{u^{k}}$. The Newton iteration is terminated if the nonlinear residual drop is sufficiently small:

$$
\left\|\frac{F\left(u^{k}\right)}{F\left(u^{0}\right)}\right\|<\text { tolerance; }
$$

or a sufficient small Newton update:

$$
\left\|\frac{\delta u^{k}}{u^{k}}\right\|<\text { tolerance; }
$$

For a scalar problem with $\mathrm{n}$ equations and $\mathrm{n}$ unknowns, we have $F(u)=\left\{F_{1}, F_{2}, \ldots F_{n}\right\}$ and $u=$ $\left\{u_{1}, u_{2}, \ldots u_{n}\right\}$, the $(i, j)$ th element of the Jacobian matrix is

$$
J_{i j}=\frac{\partial F_{i}(u)}{\partial u_{j}} \text {. }
$$

In JFNK approach, a Krylov method is used to solve the linear system of equations given by Eq. (16). An initial linear residual, $r_{0}$, is defined, given an initial guess, $\delta u_{0}$, for the Newton correction,

$$
r_{0}=-F(u)-J(u) \delta u
$$

Note that the nonlinear iteration index $k$ has been dropped since the Krylov iteration is performed at a fixed $k$. Let $j$ be the Krylov iteration index, the $j$ th iteration $\delta u_{j}$ is drawn from the subspace spanned by the Krylov vectors, $\left\{r_{0}, J r_{0}, J^{2} r_{0}, \ldots, J^{j-1} r_{0}\right\}$, and can be written as

$$
\delta u_{j}=\delta u_{0}+\sum_{i=0}^{j-1} \beta_{i}(J)^{i} r_{0}
$$

where the scalars $\beta_{i}$ minimize the residuals.

The JFNK method does not require the formation of the Jacobian matrix, instead it only needs the result vector that approximates this matrix multiplied by a vector:

$$
J v \approx \frac{[F(u+\epsilon v)-F(u)]}{\epsilon},
$$

where $\epsilon$ is a small perturbation. 
However, in most applications, the Krylov subspace methods require preconditioning to be efficient. Using right preconditioning, Eq. (16) becomes

$$
J(u) P^{-1} P \delta u=-F(u),
$$

in which $P$ represents the precondition matrix (or process) and $P^{-1}$ the inverse of preconditioning matrix. In the solution methods currently used in SAM, an analytical Jacobian matrix is computed according to Eq. (9), and passed to the underlying numerical solver library (PETSc) as the matrix $P$ for the preconditioning purpose. A very good review of the JFNK method can be found in Ref. [17].

\section{DEMONSTRATION TESTS}

\subsection{The Effects of Spatial Discretization Scheme}

As discussed above, the $2^{\text {nd }}$ order FE shape functions and mesh are supported for high efficiency and high code performance in SAM. Here, a core channel problem (coolant flow and solid conduction in fuel assembly) with uniform power distribution inside the fuel pin is presented to confirm its efficiency.

The schematic of the spatial discretization of the core channel problem is shown in Figure 1. The different lines of colors on the left represent different heat structures in an SFR fuel pin (i.e., fuel, sodium gap, and clad). Note that each element between two nodes represents a first-order 1-D finite element. If an extra node is added in the center of the element, it becomes a second-order element. The fluid and solid domains exchange energy at the fluid-structure interface nodes. A more detailed discussion of the conjugate heat transfer modeling in SAM can be found in Ref. [14]. The inlet of the core channel flow is fixed at constant temperature and flow rate. Constant material thermophysical properties are assumed for this verification test. Therefore, the analytical solutions of this test problem can be easily derived, with coolant temperature:

$$
T_{\text {coolant }}(z)=T_{\text {in }}+\frac{q^{\prime}}{\dot{m} C_{p}} z
$$

and the fuel centerline temperature:

$$
T_{f_{-} c l}(z)=T_{i n}+q^{\prime}\left(\frac{z}{\dot{m} c_{p}}+\frac{1}{2 \pi R_{c o} h_{c}}+\frac{1}{2 \pi k_{c}} \ln \left(\frac{R_{c o}}{R_{c i}}\right)+\frac{1}{2 \pi R_{f} h_{g}}+\frac{1}{4 \pi k_{f}}\right)
$$

Both $1^{\text {st }}$ order element and $2^{\text {nd }}$ order element schemes were applied for this test problem. The errors between the code predictions and the analytical solutions are shown in Figure 2 and Figure 3 for fuel centerline temperatures and coolant temperatures, respectively. It is clearly seen that the errors from $2^{\text {nd }}$ order elements simulation are essentially zero, even though only two radial elements were used for the fuel pellet region. However, the errors from $1^{\text {st }}$ order element simulation remained notable when using 20 radial elements to model the fuel pellet. 


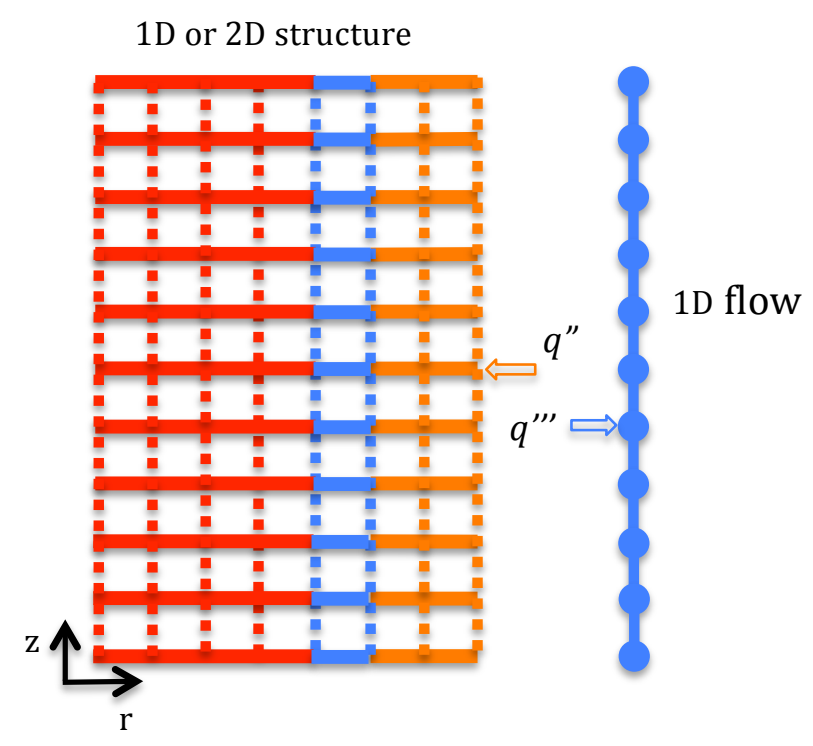

Figure 1: The schematic of the spatial discretization of the core channel problem

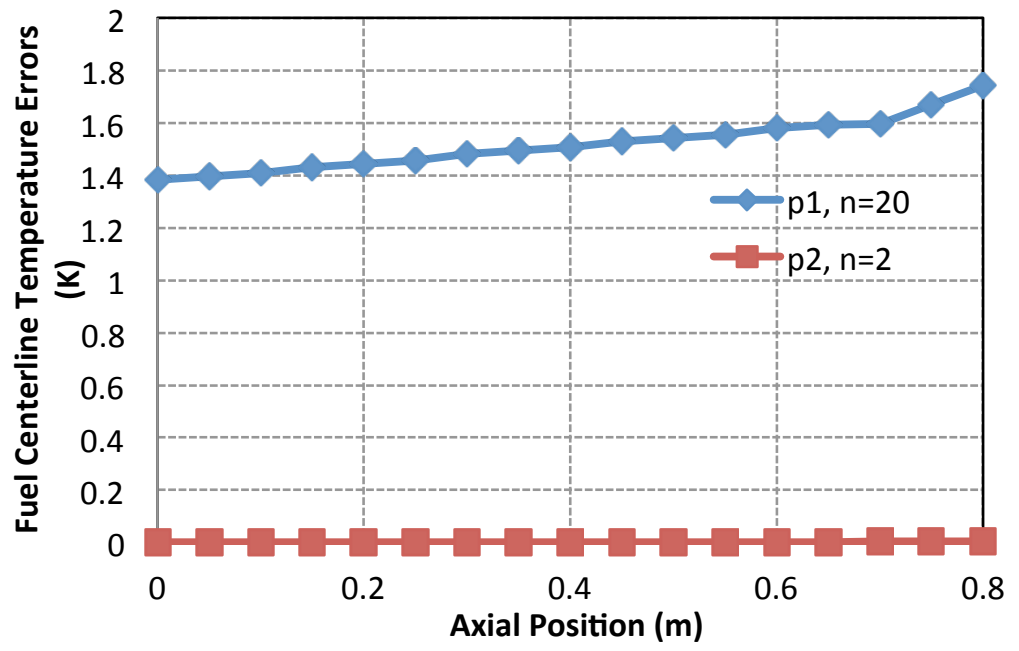

Figure 2: Errors of fuel centerline temperature predictions of a fuel assembly

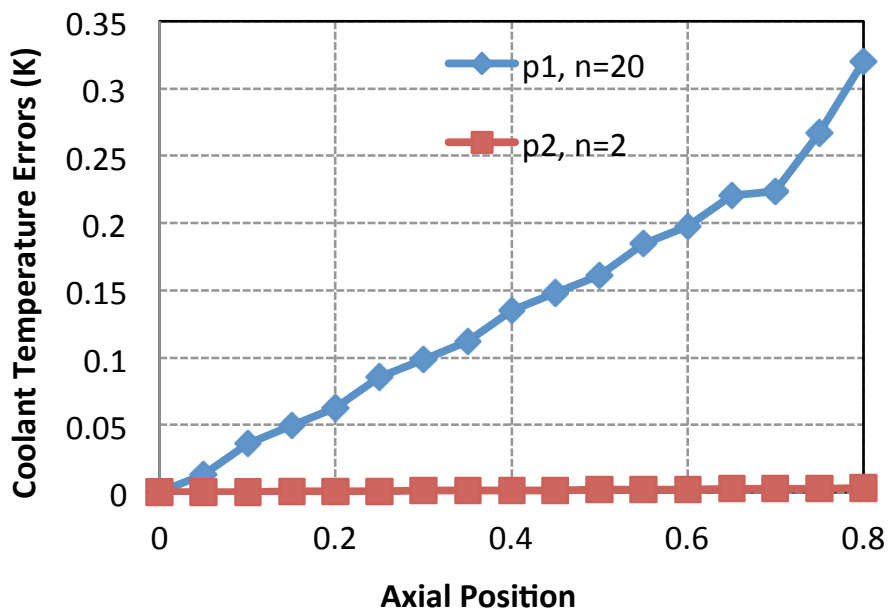


Figure 3: Errors of coolant temperature predictions of a fuel assembly

Another test problem is also examined to verify the efficiency of using $2^{\text {nd }}$ finite-elements. A solid cylinder ( $2 \mathrm{~cm}$ diameter) is heated with uniform volumetric power density inside. A constant temperature is assumed on the outer surface. The analytical radial temperature distribution can be easily derived as a quadratic function since constant material thermophysical properties are assumed.

$$
T(r)=T_{0}+\frac{q_{v^{\prime \prime \prime}}\left(r_{o}^{2}-r^{2}\right)}{4 k}
$$

In which $T_{0}$ is the outer surface temperature, and $r_{O}$ is the radius of the cylinder. Again, both $1^{\text {st }}$ order elements and $2^{\text {nd }}$ order elements were applied for this test problem. The radial temperature distributions from various spatial discretizations are shown in Figure 4. It is seen that errors still exists with 40 radial elements if using $1^{\text {st }}$ order shape function, while no errors were observed even with a single radial element if using $2^{\text {nd }}$ order shape function.

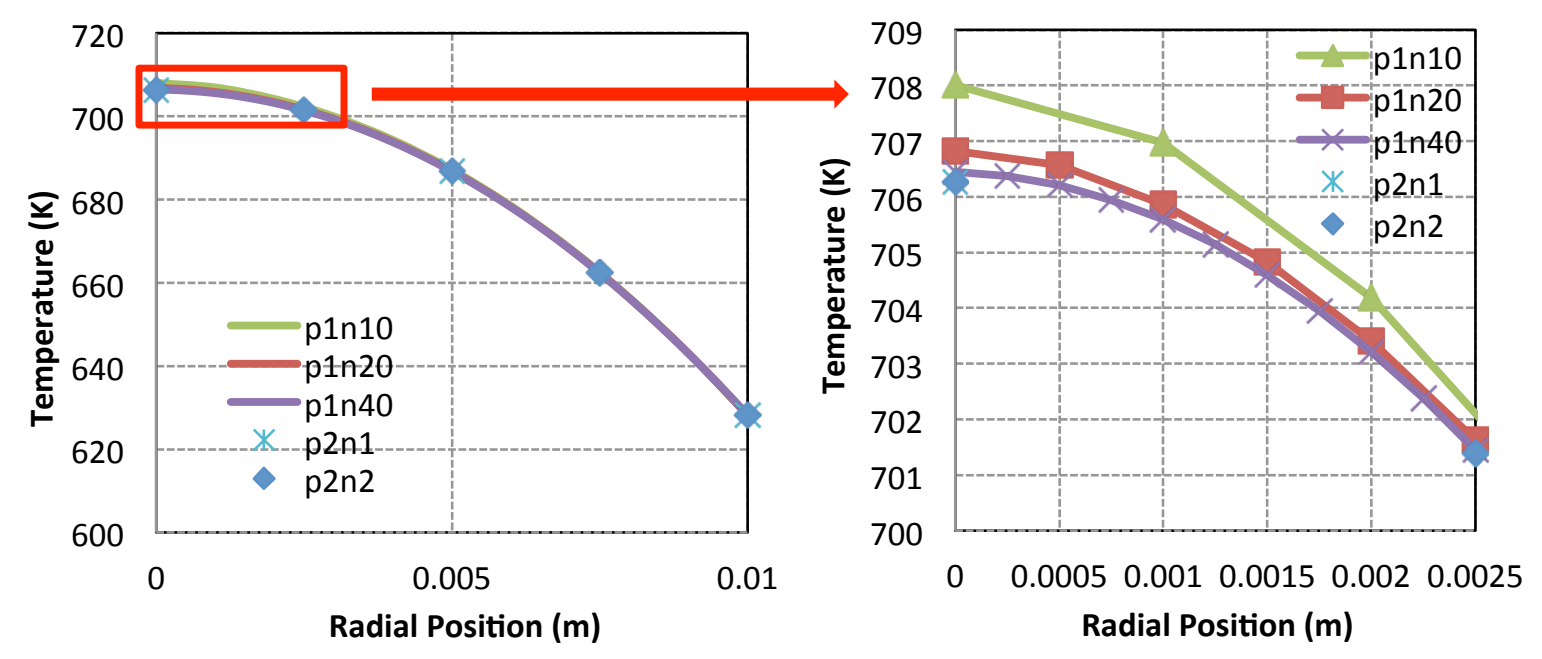

Figure 4: Radial temperature distributions of a heated pin rod

\subsection{The Effects of Temporal Discretization Scheme}

One challenging problem for traditional system codes, such as TRACE and RELAP-5, is to accurately model the wave oscillation or the sudden disturbance of the system without any numerical instability and numerical diffusion concerns due to their first-order approximations of the differential equations in both time and space. An example of the density wave propagation is presented here in a pipe flow problem. The inlet temperature of a one-meter pipe oscillates following a sinusoidal distribution, $T_{\text {in }}(t)=628+$ $100 * \sin (\pi t)$; the inlet velocity is fixed, $u_{i n}(t)=0.5 \mathrm{~m} / \mathrm{s}$; and the initial pipe temperate is at $628 \mathrm{~K}$. The transient responses of the wave propagation are shown in Figure 5, where the code predictions agreed very well with the analytical solutions. This is because of the high-order accuracy in both spatial and temporal (BDF2) discretizations in SAM. If the first-order time integration scheme (backward Euler) were used, numerical damping or diffusion would occur, as shown in Figure 6.

Another challenging problem, generally true for all types of numerical analyses, is the modeling of the non-continuity (steep gradient). A steep gradient problem is tested again in a simple pipe flow. The inlet temperature of the pipe follows a step function, and the inlet velocity is fixed $u_{\text {in }}(t)=1 \mathrm{~m} / \mathrm{s}$.

$$
T_{\text {in }}(t)=\left\{\begin{array}{l}
628 K, \text { if } t \leq 0 \\
728 K, \text { if } t>0
\end{array}\right.
$$


The transient responses of the temperature step change are shown in Figure 7, in which results from both backward Euler (BDF1) and BDF2 schemes are included. The smoothing of the temperature gradient over time is clearly observed in both schemes. The overshooting of temperature predictions was resulted at the jump with the BDF2 scheme. This is a known issue for the BDF2 scheme to model the steep gradient problems. One the other hand, the Backward Euler scheme requires much smaller time step sizes $(d t=0.001 s)$ to achieve similar diffusion comparing to the BDF2 scheme $(d t=0.01 s)$ for this test problem. Therefore, the BDF2 seems to be the better choice if efficiency is more important, while the backward Euler would be better if accuracy is more important for steep gradient problems. It should be also noted that the smoothing could be acceptable since: (1) some physical diffusions (molecule diffusion, turbulence, conduction) are real, but commonly neglected in the 1-D flow formulation; and 2) further refining the mesh and reducing the time-step size would reduce the smoothing or damping.

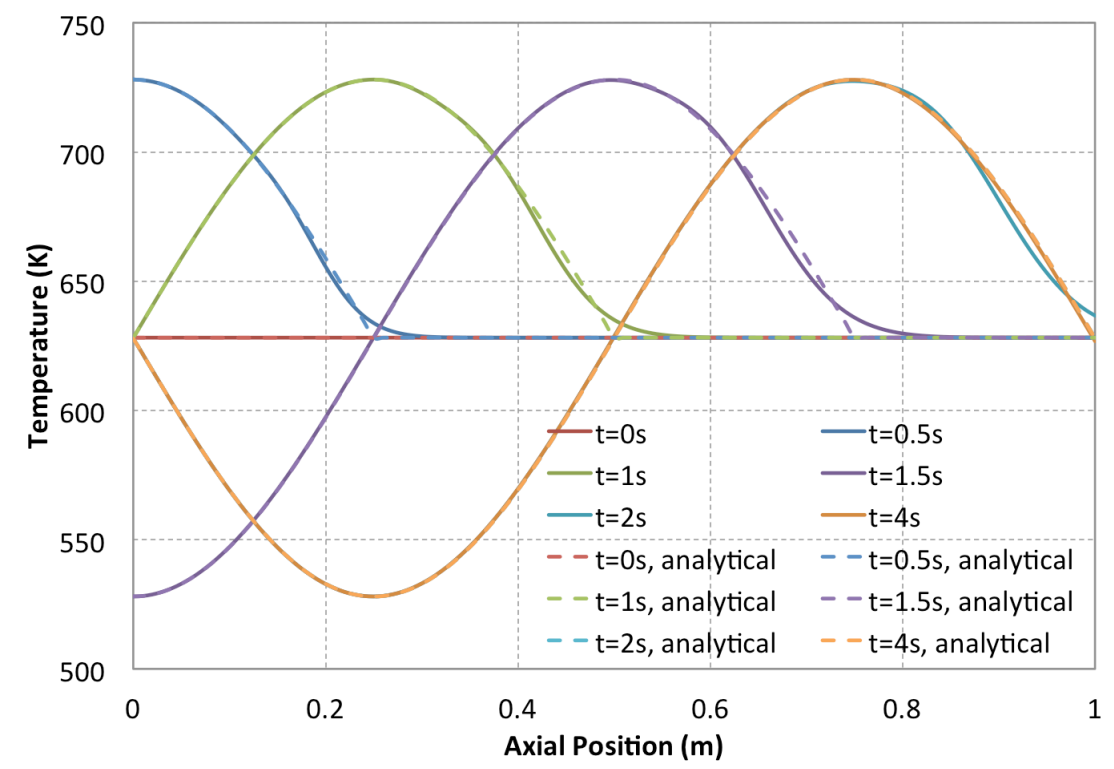

Figure 5: Transient responses of the pipe under inlet temperature oscillation, BDF2

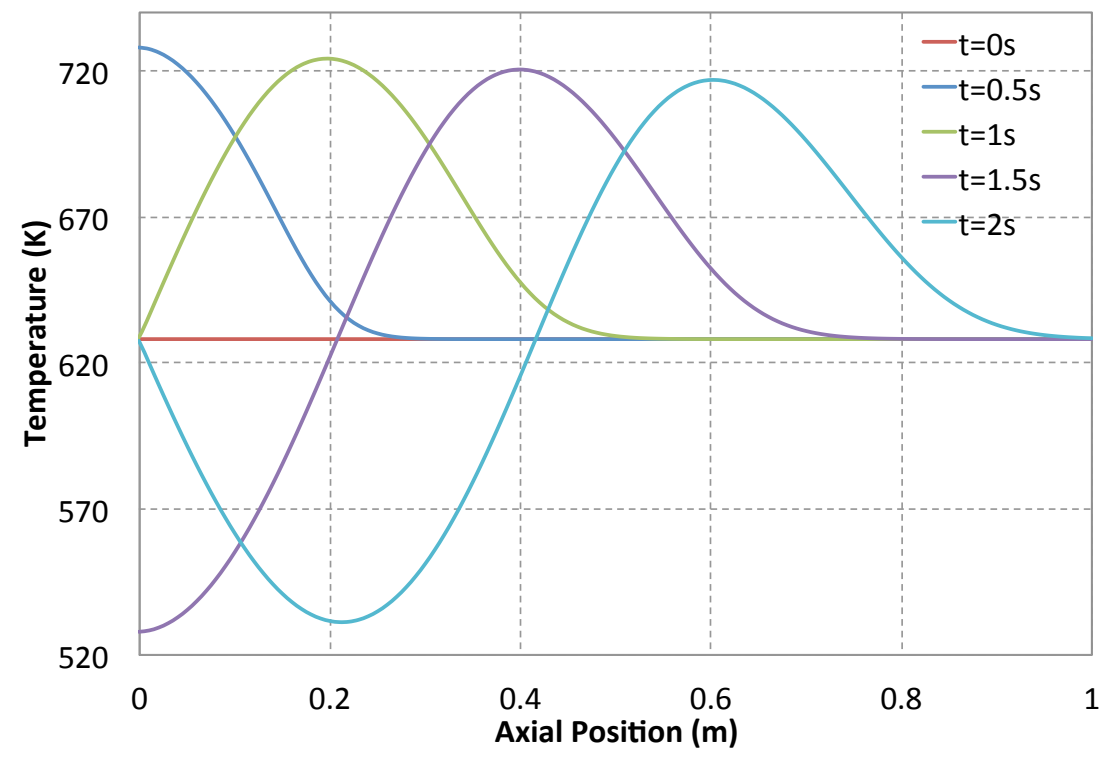

Figure 6: Damped temperature wave of the pipe under inlet temperature oscillation, backward Euler 


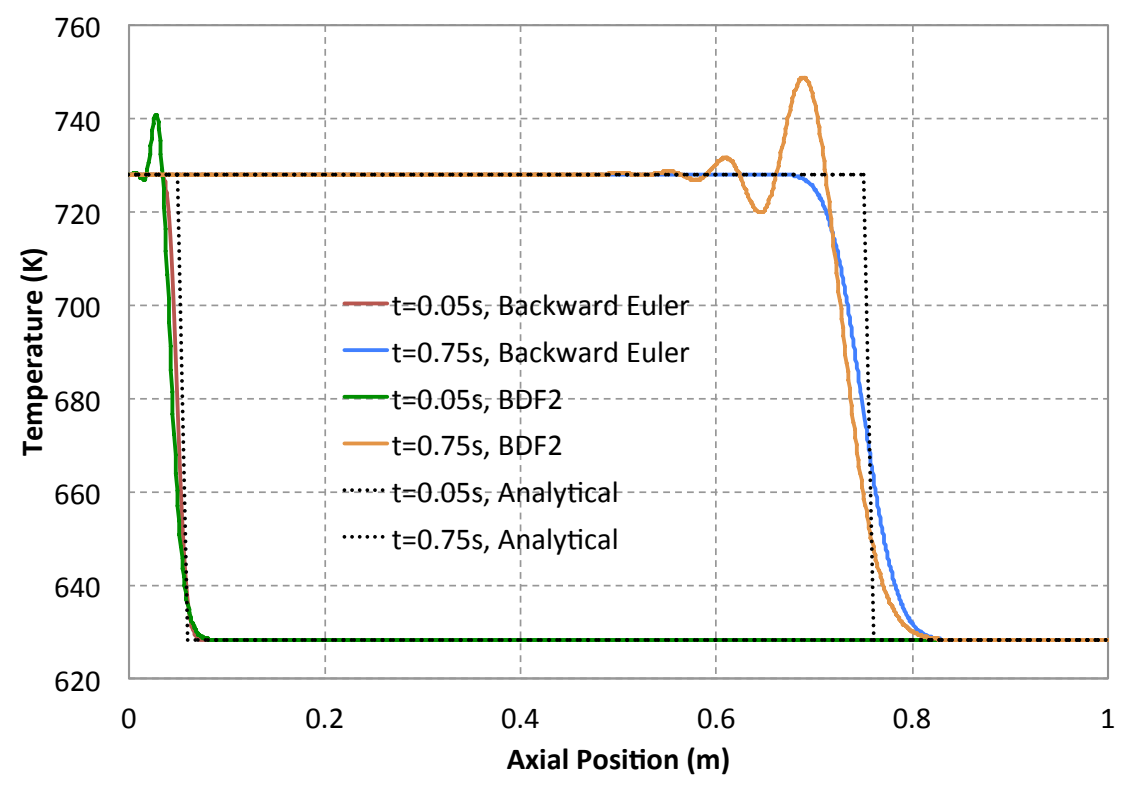

Figure 7: Smoothing of steep gradient during a temperature step change transient

\subsection{Convergence Verification Tests}

Verification of the numerical convergence rates is an essential part of the modern software verification and validation process [18]. To verify the accuracy of the SAM code on spatial discretization, a series of tests are presented here on the natural convection cooling of a used fuel assembly. In this test problem, the used SFR fuel assembly sits in a large sodium pool, and the decay heat level is assumed to be $0.4 \%$ ( 48 hours after reactor shutdown) of the peak fuel assembly in ABTR [19]. Equal pressure boundary conditions $\left(P_{0}=1 e 5 \mathrm{~Pa}\right)$ are assumed at the inside and outside of the top of the fuel assembly, as seen in Figure 8 .

Both $1^{\text {st }}$ order elements and $2^{\text {nd }}$ order elements were applied for this test problem. The errors in the predicted steady-state natural circulation flow rates from various spatial discretizations are shown in Figure 9. Since the analytical solution is very difficult to obtain, the result of the case using $402^{\text {nd }}$ order elements for each fluid component was used as the reference solution. The second order accuracy in spatial discretization is clearly demonstrated from the error trendline for the cases using $1^{\text {st }}$ order elements. The accuracy of $2^{\text {nd }}$ order elements is more difficult to obtain, since the results is already very accurate with the coarsest spatial representations ( 5 elements per fluid component), and the errors due to the settings of convergence criteria would interfere the errors due to spatial discretization.

To verify the accuracy of the SAM code on temporal discretization, the above test problem was slightly modified. The inlet pressure of the downward flow outside the assembly was assumed at a slightly higher pressure (like a pressure head provided by a pump) at steady state, $P_{i}=1.1 \mathrm{e} 5 \mathrm{~Pa}$. At $t=1 \mathrm{~s}$, the pressure is suddenly reduced to the assembly outlet pressure $\left(10^{5} \mathrm{~Pa}\right)$. This transient simulates the transition from forced flow to natural circulation flow in cooling the used fuel assembly. In this study of temporal convergence, the spatial discretization scheme of $402^{\text {nd }}$ order elements for each fluid component is used. The transient responses of core flow rates and peak clad temperatures (PCT) are shown in Figure 10. It is seen that the system approached the final steady state of natural circulation cooling after 200 seconds. The errors in the predicted PCTs from various time step sizes using the BDF2 scheme are shown in Figure 11. The result of the case using the smallest time step size $(0.2 \mathrm{~s})$ was used as the reference solution since the 
analytical solution is not available. The convergence rate of the time step size is seen about $2^{\text {nd }}$ order from the trendline.

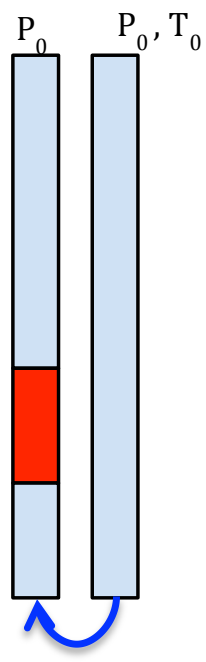

Figure 8: Schematic model of the used fuel assembly cooling test problem

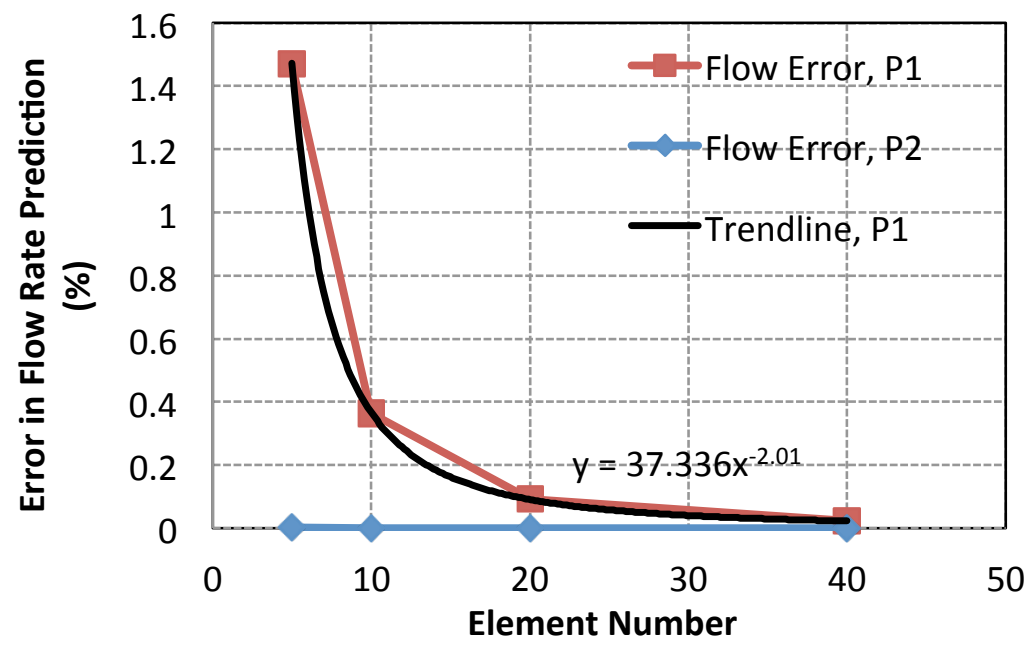

Figure 9: Spatial convergence for steady state natural circulation flow rate

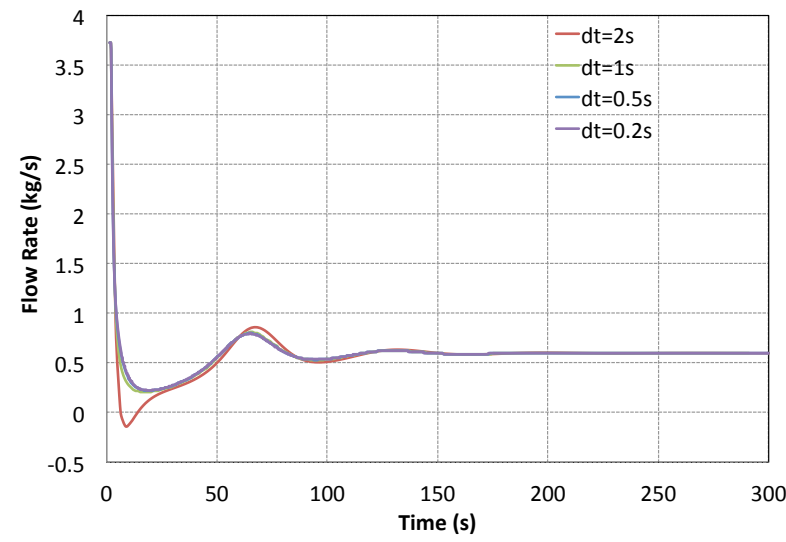

(a) Core flow rate

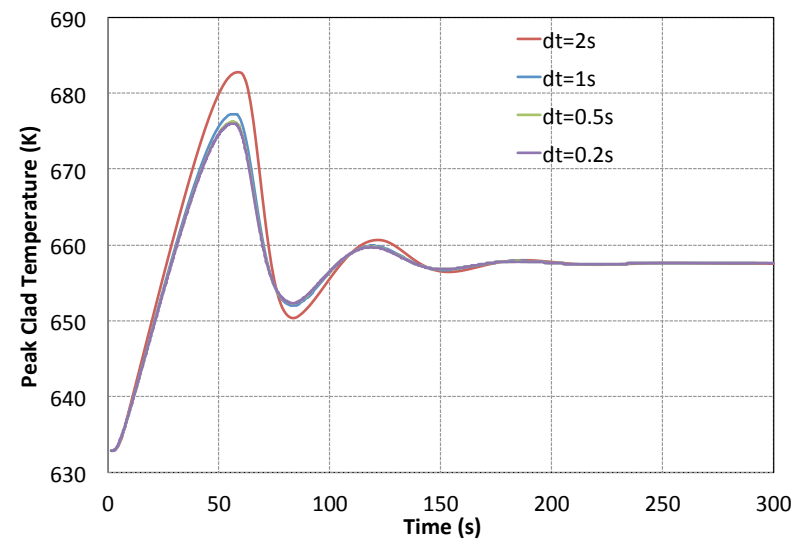

(b) Peak Clad Temperature

Figure 10: Transient response of the system from forced low to natural circulation, time step effects 


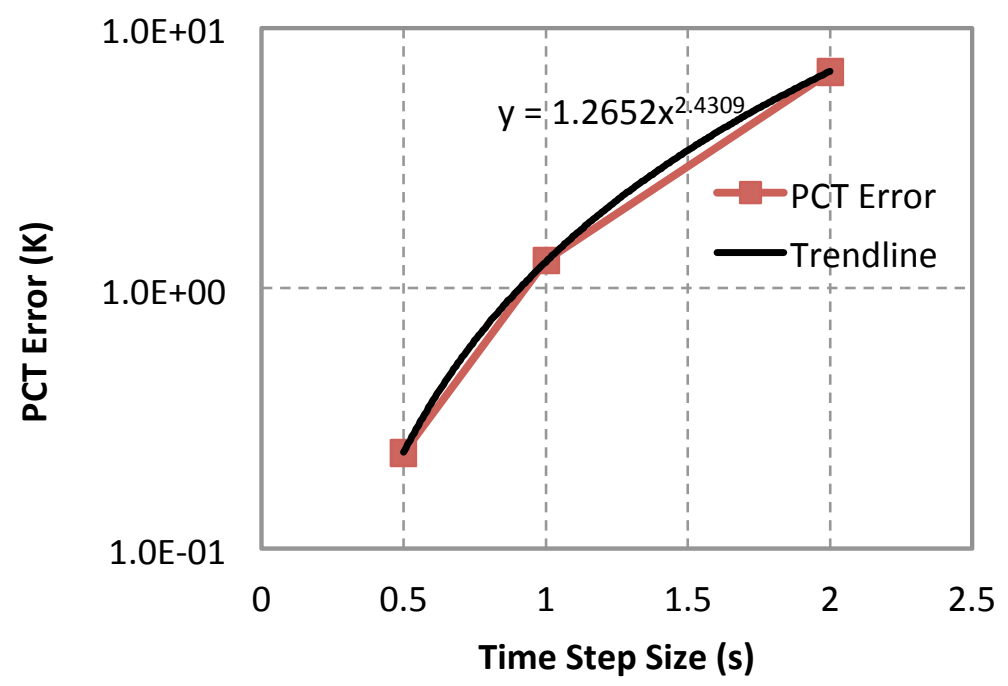

Figure 11: Time convergence of the peak clad temperature in the transient cooling test

\section{CONCLUSIONS}

An advanced system thermal-hydraulics analysis tool is currently under development for advanced reactor systems. A 1-D FEM flow model with stabilization schemes has been developed for incompressible but thermally expandable flows. High order spatial and high-order time discretization schemes have been applied to solve the one-dimensional fluid flow and heat transfer. Combined with the use of the JFNK solution method and high-order discretization schemes, this STH model enables the high efficiency and code performance, accuracy, as well as assuring the conservation of conserved quantities in the system analysis of advanced reactors.

The effects of different spatial and temporal discretization schemes are investigated. It is found that the use of $2^{\text {nd }}$ order finite elements would significantly increase the efficiency and accuracy of the simulations. The BDF2 scheme is generally preferred for its second-order accuracy and minimal numerical diffusion for continuous problems; however, backward Euler scheme could be preferred to avoid potential overshooting and undershooting for steep gradient (or discontinuous) problems. Additionally, the convergence rates of the high-order spatial and temporal discretization schemes have been confirmed by a series of verification tests. It can be concluded that the developed system thermalhydraulics model can be strictly verified, and that it performs very well for a wide range of flow problems with high accuracy, efficiency, and minimal numerical diffusions.

\section{ACKNOWLEDGMENTS}

This work is supported by U.S. DOE Office of Nuclear Energy's Nuclear Energy Advanced Modeling and Simulation (NEAMS) program. The submitted manuscript has been created by UChicago Argonne, LLC, Operator of Argonne National Laboratory ("Argonne"). Argonne, a U.S. Department of Energy Office of Science laboratory, is operated under Contract No. DE-AC02-06CH11357. 


\section{REFERENCES}

1. F. D'Auria, "Perspectives in System Thermal-Hydraulics," Nuclear Engineering and Technology, Vol. 44 (8), 855-870, (2012).

2. R.A. Berry, J.W. Peterson, H. Zhang, et al., "RELAP-7 Theory Manual," INL/EXT-14-31366, Idaho National Laboratory, December 2014.

3. P. Emonot, A. Souyri, J. L. Gandrille, and F. Barré, "CATHARE-3: A new system code for thermalhydraulics in the context of the NEPTUNE project." Nuclear Engineering and Design, 241(11), 4476-4481, (2011).

4. D. Wang, J.H. Mahaffy, J. Staudenmeier, C.G. Thurstonb, "Implementation and Assessment of HighResolution Numerical Methods in TRACE," Nuclear Engineering and Design, 263, 327-341, (2013).

5. V. A. Mousseau, "A Fully Implicit, Second Order in Time, Simulation of a Nuclear Reactor Core", Proceedings of ICONE-14, Miami, Florida, USA, July 17-20, 2006.

6. L. Zou, H. Zhao, H. Zhang, "Applications of high-resolution spatial discretization scheme and Jacobian-free Newton-Krylov method in two-phase flow problems," Annals of Nuclear Energy, Vol. 83, 101-107, (2015).

7. R. Hu and T.H. Fanning, "Update on Developments for the SFR System Analysis Module," ANL/NE-14/9, Argonne National Laboratory, September 2014.

8. R. Hu, T.H. Fanning, T. Sumner, Y. Yu, "Status Report on NEAMS System Analysis Module Development," ANL/NE-15/41, Argonne National Laboratory, December 2015.

9. D. Gaston, C. Newman, G. Hansen, and D. Lebrun-Grandi'e, "MOOSE: A parallel computational framework for coupled systems of nonlinear equations," Nuclear Engineering and Design, vol. 239, pp. 1768-1778, (2009).

10. B. S. Kirk, J. W. Peterson, R. H. Stogner, and G. F. Carey, "libMesh: A C++ Library for Parallel Adaptive Mesh Refinement/Coarsening Simulations," Engineering with Computers, 22(3-4): 237254, (2006).

11. S. Balay, J. Brown, et al., PETSc Web page, http://www.mcs.anl.gov/petsc, 2015.

12. R. Hu, "An Advanced One-Dimensional Finite Element Model for Incompressible Thermally Expandable Flow", Nuclear Technology, Vol. 190, No. 3, 313-322, (2015).

13. T.E. Tezduyar, "Stabilized Finite Element Formulations for Incompressible Flow Computations," Advances in Applied Mechanics, Vol. 28, 1-44, (1992).

14. R. Hu and Y. Yu, "Pseudo 3D Full-Core Conjugate Heat Transfer Modeling in Sodium Fast Reactors", Proceedings of NURETH-16, Chicago IL, USA, August 30-September 3, 2015.

15. F. Shakib, "Finite Element Analysis of the Compressible Euler and Navier-Stokes Equations," Ph.D. Thesis, Stanford University, (1988).

16. D. Kahaner, C. Moler, and S. Nash, Numerical Methods and Software, Prentice-Hall, New Jersey, USA, 1989.

17. D. A. Knoll and D. E. Keyes, "Jacobian-free Newton-Krylov Methods: a Survey of Approaches and Applications," Journal of Computational Physics, Vol. 193, pp.357-397 (2004).

18. H. Zhao, L. Zou, H. Zhang, et al., "Numerical Verification of the RELAP-7 Core Channel SinglePhase Model," Proceedings of ATH'14, Reno, Nevada, June 15-19, 2014.

19. Y. I. Chang, P. J. Finck, C. Grandy, et al., "Advanced Burner Test Reactor Preconceptual Design Report,” ANL-ABR-1 (ANL-AFCI-173), Argonne National Laboratory, September 2006. 